\section{Sex differences in long-term outcomes of patients with percutaneous coronary intervention: the Armenian experience}

\author{
Yeva Sahakyan, ${ }^{1,2}$ Michael E. Thompson,, \\ Lusine Abrahamyan ${ }^{1,4}$ \\ ${ }^{1}$ College of Health Sciences, American \\ University of Armenia, Yerevan; \\ 'Department of Therapy, Yerevan State \\ Medical University, Yerevan, Armenia; \\ ${ }^{3}$ Department of Public Health Sciences, \\ The University of North Carolina at \\ Charlotte, NC, USA; ${ }^{4}$ Toronto Health \\ Economics and Technology Assessment \\ (THETA) Collaborative, University of \\ Toronto, Ontario, Canada
}

\section{Abstract}

The present study aimed at assessing sex differences in perioperative characteristics and 3-year event-free survival from major adverse cardiac and cerebrovascular events (MACCE) in patients with percutaneous coronary intervention (PCI) in Armenia. The study utilized an observational, retrospective cohort design enrolling patients who underwent PCI from 2006 to 2008 at a single center in Yerevan, Armenia. Major adverse cardiac and cerebrovascular events included all-cause mortality, myocardial infarction (MI), repeat revascularization, or stroke/transient ischemic attack. Among 485 participants included in the analysis, 419 (86\%) were men. Women were older, more hypertensive, more obese, and had significantly higher rates of diabetes. At the end of follow-up, the incidence of MACCE was $37 \%$ for men and $33 \%$ for women $(\mathrm{P}=0.9)$. Based on the results from the adjusted Cox proportional hazards model, the independent predictors of MACCE included acute MI [hazard ratio $(\mathrm{HR})=1.43,95 \%$ confidence interval (CI): 1.02-2.00], arrhythmia (HR=1.64, 95\% CI: $1.07-2.50)$, sex (HR=2.46, 95\% CI: $1.08-$ 5.61), diabetes ( $\mathrm{HR}=5.65,95 \% \mathrm{CI}$ : 2.14-14.95), and the interaction between sex and diabetes ( $\mathrm{HR}=0.16$; 95\% CI: 0.05-0.47). Among diabetic patients, men had better event-free survival from MACCE (HR=0.40, 95\% CI: 0.19-0.85) than women, whereas in patients without diabetes men had worse outcomes than women (95\% CI: 1.08-5.62). In Armenia, the baseline profile of women undergoing PCI differed considerably from that of men. In patients with diabetes, women had worse outcomes at longterm follow-up, while the opposite was noted in patients without diabetes.

\section{Introduction}

Coronary artery disease (CAD) is the leading cause of morbidity and mortality among both men and women worldwide. ${ }^{1}$ Although historically considered a man's disease because of its earlier manifestation in a man's life, recent studies have indicated that more females die from CAD than males. ${ }^{2,3}$ Nonetheless, women are referred less frequently for invasive interventions such as percutaneous coronary intervention (PCI), comprising only one-third of all PCIs performed in the US. ${ }^{4}$ Such a discrepancy may be explained by the belief that women do not benefit from invasive strategies as much as men do. ${ }^{2}$ Studies have documented that women have worse clinical outcomes such as myocardial infarction (MI), stroke, and vascular complication after PCI than men. ${ }^{2,5}$ Poor outcomes can be attributed to a higher prevalence of risk factors and comorbidities such as older age, obesity, hypertension, diabetes mellitus, and congestive heart failure in women than in men at the time of the intervention. ${ }^{2,5,6}$ After adjustment for these factors, several studies reported a persistent survival disadvantage for women. ${ }^{2,4}$ Several other studies, however, reported that the sex differences disappeared after adjustments ${ }^{5-9}$ or that women had better outcomes. $^{10-12}$

Armenia, located in the Caucasus, has a population of approximately 3 million people. ${ }^{13}$ The burden of CAD in Armenia is significant. According to Armenia's Ministry of Health, in 2009 CAD morbidity was 1967/100,000 and mortality was $247 / 100,000$ population. ${ }^{14}$ Given the conflicting evidence on gender differences and the paucity of information on CAD in Armenia, this study assessed sex differences in the long-term clinical outcomes of PCI patients in Armenia treated at the Nork Marash Medical Center (NMMC) in Yerevan. The Nork Marash Medical Center is the largest tertiary cardiac surgery center in Armenia and boasts outcomes comparable to those observed in other international cardiac centers. ${ }^{15}$ In this study we evaluated sex differences in average patient-reported 3-year event-free survival from the composite major adverse cardiac and cerebrovascular events (MACCE) in patients with CAD who had PCI at NMMC.

\section{Materials and Methods}

The study utilized an observational, retrospective cohort design. The sample included all patients with $\mathrm{CAD}$ who had undergone PCI at NMMC from 1 January 2006 to 31 December 2008. Patients with missing contact information, missing medical records, residing outside
Correspondence: Lusine Abrahamyan, Toronto Health Economics and Technology Assessment (THETA) Collaborative, University of Toronto, 144 College Street, ON M5S 2S2 Toronto, Ontario, Canada.

Tel. +1.416.946.3718 - Fax: +1.416.946.3719.

E-mail: lusine.abrahamyan@utoronto.ca

Key words: percutaneous coronary intervention, diabetes, long-term outcomes, Armenia.

Contributions: YS developed the project proposal, acquired data, performed data analysis and wrote the draft paper; LA and MET provided guidance to project proposal, data analysis and interpretation, and critically revised the manuscript.

Conflict of interests: the authors declare no potential conflict of interests.

Funding: this study was made possible by the internal student support funds provided by the College of Health Sciences and the Center for Health Services Research and Development of the American University of Armenia.

Received for publication: 15 February 2013.

Revision received: 14 April 2013.

Accepted for publication: 15 April 2013.

This work is licensed under a Creative Commons Attribution 3.0 License (by-nc 3.0).

(c) Copyright Y. Sahakyan et al., 2013

Licensee PAGEPress, Italy

Healthcare in Low-resource Settings 2013; 1:e17 doi:10.4081/hls.2013.e17

of Armenia at the time of the study, or who did not speak Armenian were excluded. Patient contact information was abstracted from the NMMC PCI dataset. Telephone surveys were conducted from February to April 2011 to evaluate patients' long-term outcomes and to obtain consent to review patients' medical records for perioperative information. The study protocol was approved by the Institutional Review Board at the American University of Armenia and by the NMMC Administrative Board.

The main outcome of interest was the 3-year average survival rate from the composite MACCE that included all-cause mortality, MI, repeat revascularization, or stroke/transient ischemic attack (TIA) established by patient self-reports. All patient-reported repeat hospitalizations to NMMC were verified using the NMMC database. We also assessed patients' hospital length of stay, prescription of discharge medications, and in-hospital and early operative complications and mortality. A repeat revascularization was defined as a repeat surgical (coronary artery bypass grafting) or percutaneous coronary intervention (target or new vessel). Operative complications were defined as all major events occurring within 30 days after the PCI. 


\section{Statistical analysis}

Continuous variables are presented as means and standard deviations and compared between groups using Student's $t$-test; categorical variables are presented as counts and percentages and compared using a chi-square test or Fisher's exact test. The event-free survival rate from MACCE was estimated by the Kaplan-Meier product-limit method. Cox proportional hazard models were used to estimate unadjusted and adjusted hazard ratios (HR) of MACCE at the end of follow-up. Backward stepwise elimination was used for the multivariable Cox proportional hazards model. All variables found significant in univariate analyses $(\mathrm{P}<0.05)$ and those found predictive from past studies were entered together into the model at once and eliminated using the likelihood ratio test. The final model was checked for effect modifiers and for conformity with the proportionality assumption. All statistical analyses were performed using the Stata10 software package (Stata Statistical Software, College Station, TX, USA).

\section{Results}

Overall, 894 patients underwent PCI from 2006 to 2008 at NMMC. Of these, 839 were residents of Armenia. We could not reach 315 patients for various reasons (i.e. phone number not provided, wrong number/number changed, patient was out of the country at the time of contact, no response to call). In total, 524 patients were contacted for the phone interview. Of these, 23 refused to participate, 3 were found to be ineligible, and, after the interviews, medical records were not available for 13 patients. If the patient was reported as dead $(n=38)$ at the time of the interview, information about the MACCE and consent to access the medical records was obtained from an immediate family member. The final total sample included 485 patients.

\section{Patient baseline and procedural characteristics}

The study sample $(\mathrm{n}=485)$ included 419 (86\%) men and 66 women. Patients' baseline characteristics stratified by sex are presented in Table 1 . Women were on average 5 years older than men and had a higher prevalence of hypertension, obesity, and diabetes and more often presented with stable angina. A significantly higher proportion of men smoked and at admission presented with acute MI more frequently than women.

No differences were observed in the number of diseased vessels and the number and types of stents implanted (Table 1). In men and women, the most frequently stented vessel was the left anterior descending (LAD) artery. No statistically significant differences were seen between women and men in discharge medication, except for a higher rate of angiotensinconverting enzyme inhibitors (ACEi)/ angiotensin receptor blockers (ARB) prescribed to women.

\section{Acute in-hospital and 30-day oper- ative outcomes}

Thirty-day operative complications were observed in 3 women (4.5\%) and in 23 men (5.5\%, $\mathrm{P}=0.7)$. Overall, the following complications were observed: ventricular tachycardia/ ventricular fibrillation $(\mathrm{n}=4)$; complete atri- oventricular block $(n=2)$; hematoma at the intervention site $(n=1)$; coronary artery dissection $(n=1)$; reperfusion syndrome $(n=1)$; stent thrombosis $(n=2)$; TIA $(n=1)$; acute renal failure $(n=1)$; acute heart failure $(n=1)$; $\mathrm{LAD}$ occlusion during coronary angiography $(\mathrm{n}=1)$; recurrent MI $(\mathrm{n}=2)$; and repeat revascularization $(n=4)$. In-hospital deaths occurred in 2 men. Death within 30 days after PCI occurred in 1 woman and 3 men. The hospital length of stay did not differ between sexes and was on average $4.5 \pm 3.6$ days for the total sample.

Table 1. Baseline patients' characteristics.

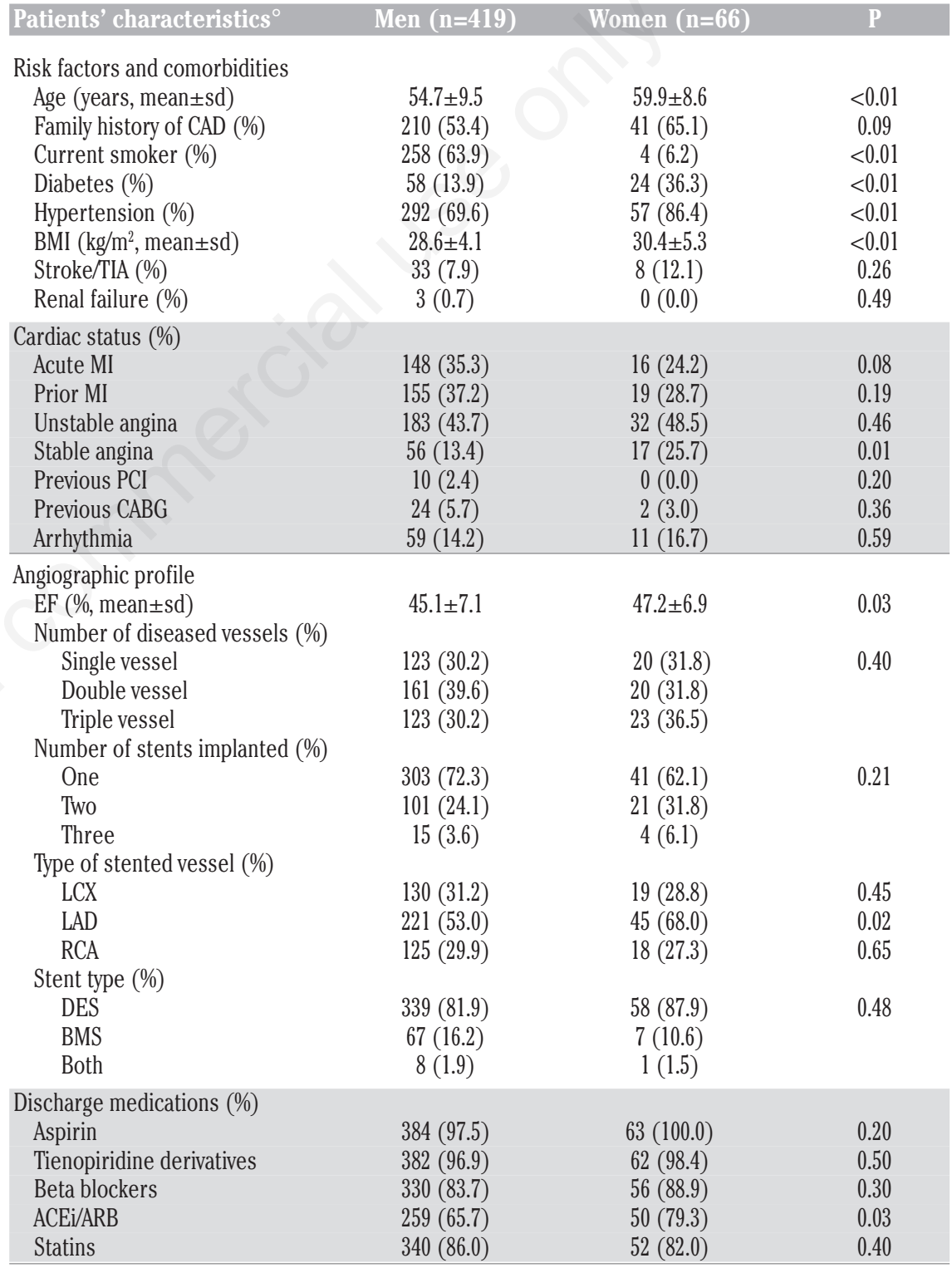

CAD, coronary artery disease; BMI, body mass index; TIA, transient ischemic attack; MI, myocardial infarction; PCI, percutaneous coronary intervention; $\mathrm{CABG}$, coronary artery bypass graft; $\mathrm{EF}$, ejection fraction; LCX, left circumflex; LAD, left anterior descending; RCA, right coronary artery; DES, drug eluting stent; BMS, bare metal stent; ACEi/ARB, angiotensin converting enzyme inhibitors/angiotensin receptor blockers. ${ }^{\circ}$ Results are presented as frequencies and percentages, unless specified otherwise. All percentages were calculated after excluding missing values. 


\section{Event-free survival rates at long-term follow-up}

The median follow-up of the total sample was 1148 days, ranging from 3 to 1917. The mean follow-up was $1267 \pm 321$ days for women and $1232 \pm 321$ days for men $(\mathrm{P}=0.4)$. During the follow-up period, the total number of MACCE $(n=180)$ did not significantly differ between men and women (37.0 vs $33.3 \%$, $\mathrm{P}=0.9$ ) (Table 2). The most frequently observed MACCE in both groups was repeat revascularization. The event-free survival from MACCE at the median follow-up was 0.79 (95\% CI: $0.66-0.87)$ for women and 0.74 (95\% CI: 0.69-0.78) for men ( $\mathrm{P}>0.05)$.

The unadjusted predictors of long-term survival (MACCE) were identified using univariate Cox proportional hazard models. Significant predictors $(\mathrm{P}<0.05)$ of event-free survival were acute MI at admission, arrhythmia, left ventricular ejection fraction, number of diseased vessels, and stent type.

The final, multivariable model included sex (HR=2.46, 95\% CI: 1.08-5.61), diabetes (HR=5.65, 95\% CI: 2.14-14.95), arrhythmia (HR=1.64, 95\% CI: 1.07-2.50), acute MI at admission (HR=1.43, 95\% CI: 1.02-2.00), and the interaction between sex and diabetes (HR=0.16; 95\% CI: 0.05-0.47) (Table 3).

In patients without diabetes, after adjusting for arrhythmia and acute MI at admission, men had worse event-free survival from MACCE (HR=2.46, 95\% CI: 1.08-5.62) than women (Table 4, Figure 1A). After adjusting for arrhythmia and acute MI at admission, in patients with diabetes, men had better eventfree survival from MACCE (HR=0.40, 95\% CI: 0.19-0.85) than women (Table 4, Figure 1B).

\section{Discussion}

This observational study sought to evaluate sex differences in 3-year event-free survival from MACCE in patients with CAD who had $\mathrm{PCI}$ in a single center in Armenia. We observed significant differences in several baseline factors between men and women. For example, women on average were older than men, more hypertensive, more obese, and had a significantly higher rate of diabetes. In contrast, men were more likely to be smokers. Similar differences were observed in several past studies. ${ }^{2,5,11,16,17}$ For example, a recent observational study conducted by Duvernoy et al. found that women were more obese (47.9 vs $43.1 \%$ ) and more often had diabetes mellitus (38.5 vs $29.2 \%$ ) and hypertension (82.5 vs $71.0 \%$ ), and that men were more likely to smoke (27.3 vs $21.7 \%)^{2}$

In the current analysis, the unadjusted event-free survival at the end of follow-up was
Table 2. Distribution of major adverse cardiac and cerebrovascular events between sexes.

\begin{tabular}{lcccc} 
Events, $\mathrm{n}(\%)$ & $\begin{array}{c}\text { Total sample } \\
(\mathrm{n}=485)\end{array}$ & $\begin{array}{c}\text { Men } \\
(\mathrm{n}=419)\end{array}$ & $\begin{array}{c}\text { Women } \\
(\mathrm{n}=66)\end{array}$ & $\mathrm{P}$ \\
$\mathrm{MI}$ & $31(6.4)$ & $26(6.2)$ & $5(7.6)$ & 0.8 \\
$\mathrm{RR}$ & $102(21.0)$ & $92(22.0)$ & $10(15.2)$ & 0.3 \\
PCI & $71(14.6)$ & $64(15.3)$ & $7(10.6)$ & \\
CABG & $32(6.6)$ & $29(6.9)$ & $3(4.5)$ & \\
\hline Death & $38(7.8)$ & $31(7.4)$ & $7(10.6)$ & 0.4 \\
Stroke/TIA & $9(1.9)$ & $9(2.2)$ & $0(0.0)$ & 0.4 \\
\hline Total MACCE & $180(37.1)$ & $158(37.7)$ & $22(33.3)$ & 0.9 \\
\hline
\end{tabular}

MI, myocardial infarction; RR, repeat revascularization; $\mathrm{PCI}$, percutaneous coronary intervention; CABG, coronary artery bypass graft; TIA, transient ischemic attack; MACCE, major adverse cardiac and cerebrovascular events.

Table 3. Unadjusted and adjusted Cox proportional hazard models of survival from major adverse cardiac and cerebrovascular events.

\begin{tabular}{lcccccc} 
& \multicolumn{3}{c}{ Unadjusted } & \multicolumn{3}{c}{ Adjusted } \\
Predictors & Havard ratio & $95 \%$ CI & P & Havard ratio & $95 \%$ CI & P \\
Sex & 1.12 & $0.69-1.81$ & 0.652 & 2.46 & $1.08-5.61$ & 0.032 \\
Diabetes & 1.28 & $0.85-1.94$ & 0.241 & 5.65 & $2.14-14.95$ & 0.000 \\
\hline Acute MI & 1.51 & $1.09-2.10$ & 0.014 & 1.43 & $1.02-2.00$ & 0.036 \\
Arrhythmia & 1.66 & $1.09-2.53$ & 0.018 & 1.64 & $1.07-2.50$ & 0.022 \\
\hline Sex*diabetes & - & - & - & 0.16 & $0.05-0.47$ & 0.001 \\
\hline
\end{tabular}

$\mathrm{CI}$, confidence interval; MI, myocardial infarction; sex*diabetes, interaction between sex and diabetes.

Table 4. Interaction between sex and diabetes in survival from major adverse cardiac and cerebrovascular events after controlling for acute myocardial infarction and arrhythmia.

\begin{tabular}{lcccc} 
& Patients (n) & MACCE (n) & Hazard ratio (95\% CI) & P \\
Diabetes & & & & \\
Male & 58 & 15 & $0.40(0.19-0.85)$ & 0.02 \\
Female & 24 & 15 & 1.0 (reference) & \\
No diabetes & & & & \\
Male & 361 & 143 & $2.46(1.08-5.61)$ & 0.03 \\
Female & 42 & 7 & 1.0 (reference) & \\
\hline
\end{tabular}

MACCE, major adverse cardiac and cerebrovascular events; Cl, confidence interval.

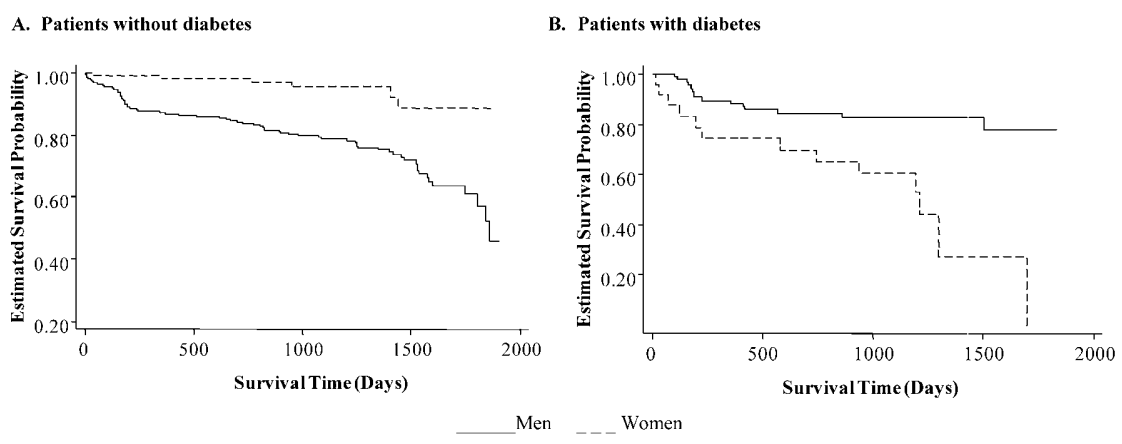

Figure 1. Survivor functions by sex, adjusted for acute myocardial infarction and arrhythmia. 
similar between sexes, despite the differences in baseline profiles. These results agree with past studies that also had a retrospective design, followed patients 3 years or longer, and similarly enrolled patients with stable and unstable angina and acute MI. ${ }^{7,9,18}$ However, these studies demonstrated that after the adjustment for baseline differences the rates of major adverse cardiac events (MACE) were no longer different between the sexes. Overall, compared to studies in the early 1990 s, recent studies have mostly shown that with improved care and technologies, the gap between men and women in complication rates and rates of MACE at long-term follow-up is disappearing. ${ }^{19}$

The evidence of the impact of diabetes status on sex differences in PCI outcomes is still contradictory. In our sample of patients, the prevalence of diabetes was almost 2.5 times higher among women than men (36 and 14\%, respectively). After adjusting for acute $\mathrm{MI}$ and arrhythmia, we found that women with diabetes had a higher risk of MACCE than men. In contrast, a recent study that enrolled only patients with diabetes in Japan found that at 4 year follow-up after PCI, the cumulative incidence of MACE was similar between the sexes, despite the fact that women had a worse baseline profile. ${ }^{20}$ Similar to our study, a significant interaction was observed by Mehilli et al., who evaluated the impact of sex on mortality after PCI in a cohort of patients with stable and unstable angina. ${ }^{12}$ They reported that diabetic women had almost twice the mortality hazard in comparison to diabetic men, whereas no significant difference was observed in mortality among the non-diabetic population.

Further analysis of our data revealed that among men, diabetes status did not significantly affect the risk of developing MACCE. Among women, diabetes was a significant predictor of MACCE after adjusting for acute MI and arrhythmia (data not shown). Our finding is supported by a recent meta-analysis of 37 studies that evaluated the risk of fatal coronary events among a diabetic population. ${ }^{21}$ It demonstrated that the rate of adverse outcomes was higher among diabetic than nondiabetic patients, but the difference was more pronounced among women than men. The study concluded that the relative risk for fatal CAD associated with diabetes is overall $50 \%$ higher in women than in men, most likely due to differences in baseline risk profiles and disparities in treatment approaches.

In our study, we did not evaluate the severity of diabetes (insulin dependent or not; effectively managed or not) that might explain the observed variability in the impact of diabetes on the outcomes by sex. A study that evaluated the effectiveness of drug-eluting stents in acute coronary syndrome patients with diabetes reported a higher prevalence of insulindependent diabetes among women compared to men. ${ }^{22}$ The sex differences in our study may also be explained by unequal access to or utilization of health care services in Armenia. The 2005 Armenian demographic and health survey found that although a higher proportion of women reported having health problems than men (13.8 vs $11.2 \%$ ), men had overall higher hospitalization rates than women (2.6 vs $2.3 \%) .^{13}$

The limitations of the study merit discussion. In the studied sample, the male female ratio was almost six to one (419 to 66), thus limiting the number of independent predictors that could have been studied and potentially their precision. Another important limitation of our study was that the follow-up data about MACCE were collected retrospectively through telephone interviews, which could introduce recall and report biases. To minimize that bias, we verified self-reported outcomes with the NMMC medical records where possible. Another source of potential bias came from inaccuracies in medical records where, for example, heart failure status and blood lipid levels were not consistently reported and were excluded from the analyses. About one-third of the patients from the original sample were unreachable, either because of inaccurate contact information or absence from the country. The comparison of these non-responders with the final study population using NMMC patient registry information indicated that nonresponders were on average 2 years younger $(\mathrm{P}<0.05)$ than the enrolled patients, and the difference was mainly attributed to the difference among the male population. Thus, the non-response bias suggests that our detected differences would probably be of a larger magnitude if the total sample had been included.

\section{Conclusions}

In conclusion, we found that in Armenia, women with CAD differed from men in several baseline risk factors and comorbidities. Women were older than men and had higher prevalence of hypertension, obesity, and diabetes. A significantly higher proportion of men smoked. The differences in the long-term outcomes of PCI between men and women were dependent on the diabetes status. Future studies should investigate the nature, extent, and causal mechanism of the excess risk of diabetes on PCI outcomes, and targeted strategies should be developed to decrease this risk and improve patient outcomes.

\section{References}

1. Roger VL, Go AS, Lloyd-Jones DM, et al.
Heart disease and stroke statistics-2011 update: a report from the American Heart Association. Circulation 2011;123:e18-e209.

2. Duvernoy CS, Smith DE, Manohar P, et al. Gender differences in adverse outcomes after contemporary percutaneous coronary intervention: an analysis from the Blue Cross Blue Shield of Michigan Cardiovascular Consortium (BMC2) percutaneous coronary intervention registry. Am Heart J 2010159:677-83.

3. Shu W, Lei W, Peng S. Recent development of ischaemic heart disease in sex difference. Postgrad Med J 2007;83:240-3.

4. Lansky AJ, Hochman JS, Ward PA, et al. Percutaneous coronary intervention and adjunctive pharmacotherapy in women: a statement for healthcare professionals from the American Heart Association. Circulation 2005;111:940-53.

5. Blomkalns AL, Chen AY, Hochman JS, et al. Gender disparities in the diagnosis and treatment of non-ST-segment elevation acute coronary syndromes: large-scale observations from the CRUSADE (Can Rapid Risk Stratification of Unstable Angina Patients Suppress Adverse Outcomes With Early Implementation of the American College of Cardiology/ American Heart Association Guidelines) National Quality Improvement Initiative. J Am Coll Cardiol 2005;45:832-7.

6. Kovacic JC, Mehran R, Karajgikar R, et al. Female gender and mortality after percutaneous coronary intervention: Results from a large registry. Catheter Cardio Inte 2011;80:514-21.

7. Onuma Y, Kukreja N, Daemen J, et al. Impact of sex on 3-year outcome after percutaneous coronary intervention using bare-metal and drug-eluting stents in previously untreated coronary artery disease: insights from the RESEARCH (RapamycinEluting Stent Evaluated at Rotterdam Cardiology Hospital) and T-SEARCH (Taxus-Stent Evaluated at Rotterdam Cardiology Hospital) registries. JACC Cardiovasc Interv 2009;2:603-10.

8. Solinas E, Nikolsky E, Lansky AJ, et al. Gender-specific outcomes after sirolimuseluting stent implantation. J Am Coll Cardiol 2007;50:2111-6.

9. Singh M, Rihal CS, Gersh BJ, et al. Mortality differences between men and women after percutaneous coronary interventions. A 25year, single-center experience. J Am Coll Cardiol 2008;51:2313-20.

10. Alfredsson J, Stenestrand U, Wallentin L, et al. Gender differences in management and outcome in non-ST-elevation acute coronary syndrome. Heart 2007;93:1357-62.

11. Berger JS, Sanborn TA, Sherman W, et al. Influence of sex on in-hospital outcomes and long-term survival after contemporary 
percutaneous coronary intervention. Am Heart J 2006;151:1026-31.

12. Mehilli J, Kastrati A, Bollwein $\mathrm{H}$, et al. Gender and restenosis after coronary artery stenting. Eur Heart J 2003;24:1523-30.

13. National Statistical Service, Armenia. Armenia demographic and health Survey 2005. Yerevan: National Statistical Service of Armenia ed.; 2006. Available from: http:/www.measuredhs.com/pubs/pdf/FR18 4/FR184.pdf

14. Republic of Armenia, Ministry of Health. Health and health care of Armenia 2009. Official annual statistical report. Yerevan: Republic of Armenia, Ministry of Health ed.: 2010.

15. Abrahamyan L, Demirchyan A, Thompson $\mathrm{ME}$, et al. Determinants of morbidity and intensive care unit stay after coronary sur- gery. Asian Cardiovasc Thorac Ann 2006;14:114-8.

16. Akhter N, Milford-Beland S, Roe MT, et al. Gender differences among patients with acute coronary syndromes undergoing percutaneous coronary intervention in the American College of Cardiology-National Cardiovascular Data Registry (ACC-NCDR). Am Heart J 2009;157:141-8.

17. El-Menyar A, Zubaid M, Rashed W, et al. Comparison of men and women with acute coronary syndrome in six Middle Eastern countries. Am J Cardiol 2009;104:1018-22.

18. D'Ascenzo F, Gonella A, Quadri G, et al. Comparison of mortality rates in women versus men presenting with ST-segment elevation myocardial infarction. Am J Cardiol 2011;107:651-4.

19. Ge JB. Gender difference in patients with acute myocardial infarction treated by primary percutaneous coronary intervention in drug-eluting stent era. Chinese Med JPeking 2010;123:776-7.

20. Ogita M, Miyauchi K, Dohi T, et al. Genderbased outcomes among patients with diabetes mellitus after percutaneous coronary intervention in the drug-eluting stent era. Int Heart J 2011;52:348-52.

21. Huxley R, Barzi F, Woodward M. Excess risk of fatal coronary heart disease associated with diabetes in men and women: metaanalysis of 37 prospective cohort studies. Brit Med J 2006;332:73-8.

22. Longo G, Gonella A, D'Ascenzo F, et al. Percutaneous drug-eluting stent implantation in diabetic patients: short and long term outcomes from an observational study. Minerva Cardioangiol 2011;59:1-7. 